\title{
CpG-DNA induces bacteria-reactive IgM enhancing phagocytic activity against Staphylococcus aureus infection
}

\author{
Te Ha Kim ${ }^{1,2}$, Dongbum Kim ${ }^{2}$, Heesu Lee ${ }^{1}$, Min Hyung Kwak ${ }^{1}$, Sangkyu Park ${ }^{3}$, Younghee Lee ${ }^{3}$ E Hyung-Joo Kwon ${ }^{1,2, *}$ \\ ${ }^{1}$ Department of Microbiology, College of Medicine, Hallym University, Chuncheon 24252, ${ }^{2}$ Center for Medical Science Research, College of \\ Medicine, Hallym University, Chuncheon 24252, ${ }^{3}$ Department of Biochemistry, College of Natural Sciences, Chungbuk National \\ University, Cheongju 28644, Korea
}

CpG-DNA triggers the proliferation and differentiation of $B$ cells which results in the increased production of antibodies. The presence of bacteria-reactive IgM in normal serum was reported; however, the relevance of CpG-DNA with the production of bacteria-reactive IgM has not been investigated. Here, we proved the function of CpG-DNA for the production of bacteria-reactive IgM. CpG-DNA administration led to increased production of bacteria-reactive IgM both in the peritoneal fluid and serum through TLR9 signaling pathway. When we stimulated B cells with CpG-DNA, production of bacteria-reactive IgM was reproduced in vitro. We established a bacteria-reactive monoclonal IgM antibody using CpG-DNA stimulated-peritoneal B cells. The monoclonal IgM antibody enhanced the phagocytic activity of RAW 264.7 cells against $S$. aureus MW2 infection. Therefore, we suggest that CpG-DNA enhances the antibacterial activity of the immune system by triggering the production of bacteria-reactive IgM. We also suggest the possible application of the antibodies for the treatment of antibiotics-resistant bacterial infections. [BMB Reports 2019; 52(11): 635-640]

\section{INTRODUCTION}

Immunoglobulin $\mathrm{M}(\operatorname{lgM})$ exists as two types: natural $\lg M$ and immune IgM (1). Natural antibodies, consisting mostly of the natural IgM, are the antibodies found in the sera of humans and mice without any exposure to antigens (2). In contrast to the natural $\lg M$, immune $\operatorname{Ig} M$ is the first antibody produced in response to antigenic stimulation (3). Both IgMs have an important role in pathogen defense, especially bacterial infection (4). Several investigators have suggested that the administration of

*Corresponding author. Tel: +82-33-248-2635; Fax: +82-33-241-3640; E-mail: hjookwon@hallym.ac.kr

https://doi.org/10.5483/BMBRep.2019.52.11.018

Received 14 January 2019, Revised 11 February 2019, Accepted 13 March 2019

Keywords: CpG-DNA, IgM, Phagocytosis, Staphylococcus aureus, TLR9
IgM in both in vitro and in vivo models leads to protection against bacterial infection, such as Streptococcus agalactiae (S. agalactiae), Escherichia coli (E. coli), Borrelia hermsii (B. hermsii), Pseudomonas aeruginosa (P. aeruginosa), and Francisella tularensis (F. tularensis) (5-9). Using IgM knock out mice, some reports have also discovered the protective role of IgM expression against bacterial infection, including Streptococcus pneumoniae (S. pneumoniae) and B. hermsii $(10,11)$.

DNA containing CpG dinucleotide motifs (CpG-DNA), which are found in bacterial DNA, stimulate Toll-like receptor 9 (TLR9) on immune cells, leading to cell proliferation and cytokine production (12). Furthermore, CpG-DNA activates B cells, producing $T$ cell-independent antibodies (13). These roles of CpG-DNA in the immune system are closely related to the prevention of bacterial infection (14-17). Investigators have reported that CpG-DNA activated dendritic cells and the secreted type- 1 cytokine, IFN- $\gamma$, have protective roles against bacterial infection in murine models $(14,15)$. The antibacterial effects of reactive oxygen species (ROS) produced by the administration of CpG-DNA in osteoblast-like cell lines have also been shown in $S$. aureus infection (16). Furthermore, it was reported that CpG-DNA treatment activated macrophages to promote phagocytosis in $S$. aureus-infected conditions through the JNK/P38 signaling pathway (17).

Previously, we reported that bacteria-reactive IgG produced by the administration of CpG-DNA enhances the antibacterial effect in murine models (18); however, the biological functions of IgM produced by CpG-DNA administration are not known. Here, we confirmed that the production of TLR9-mediated IgM occurred both in the peritoneal cavity and sera of mice and that IgM was produced in the peritoneal $\mathrm{B}$ cells in vitro. We isolated a bacteria-reactive monoclonal $\lg M$ antibody induced by CpG-DNA administration and found for the first time that the IgM enhances phagocytosis against bacterial infection.

\section{RESULTS}

IgM antibodies reactive to bacteria were generated by CpG-DNA administration through the TLR9 signaling pathway

In our previous studies (18), we confirmed that the survival of

ISSN: 1976-670X (electronic edition)

Copyright (C) 2019 by the The Korean Society for Biochemistry and Molecular Biology

(c) This is an open-access article distributed under the terms of the Creative Commons Attribution Non-Commercial License (http://creativecommons.org/licenses/by-nc/4.0) which permits unrestricted non-commercial use, distribution, and reproduction in any medium, provided the original work is properly cited. 
mice after $S$. aureus MW2 infection was enhanced by injection of CpG-DNA. Based on our observations, we assumed that bacteria-reactive IgM could be produced by the injection of CpG-DNA 1826 into the peritoneal cavity. To identify the production of IgM by the CpG-DNA treatment in mice, we harvested the peritoneal fluid and serum from the mice after intraperitoneal injection of CpG-DNA 1826 followed by intravenous injection with $S$. aureus MW2, and measured the levels of IgM reactive to $S$. aureus MW2 using the ELISA assay. When the mice were intraperitoneally injected with CpG-DNA 1826, the IgM levels were significantly increased both in the peritoneal fluid and serum, especially in the peritoneal fluid. The concentration of the IgM was decreased slightly in the peritoneal fluid and serum following the infection with $S$. aureus MW2; however, the pretreatment of CpG-DNA 1826 before $S$. aureus MW2 infection showed a significant increase of the IgM concentration (Fig. 1A and B).

To analyze the binding ability of IgM produced by the CpG-DNA administration to various bacterial species, we selected Gram-positive bacterial species (Staphylococcus and Streptococcus) and Gram-negative bacterial species (Klebsiella, Escherichia, Acinetobacter and Pseudomonas) and performed ELISA assays. The results show that the levels of each bacteria-reactive $\operatorname{lgM}$ were increased in the peritoneal fluids and serum by intraperitoneal injection of CpG-DNA 1826, and the rate of increase in the peritoneal fluids was significantly higher than that of the serum (Fig. 1C-F).

To determine whether the IgM production induced by the CpG-DNA administration was due to the activation of the TLR9 signaling pathway, we performed the same experiments using $\mathrm{BALB} / \mathrm{C} \mathrm{TLR9}^{--}$mice. There were no significant changes in IgM production both in the peritoneal fluid and sera of the TLR9 ${ }^{-/-}$mice even with the injection of CpG-DNA 1826 (Fig. 1G-J). Therefore, the results suggest that the TLR9 signaling pathway is involved in the production of bacteria-reactive IgM induced by CpG-DNA stimulation.

\section{Induction of bacterial-reactive IgM in mouse peritoneal cavity cells by CpG-DNA treatment in vitro}

To identify where the bacteria-reactive IgM was produced by the CpG-DNA administration, the mice were intraperitoneally injected with CpG-DNA 1826 or non-CpG-DNA 2041, and then the peritoneal fluid, spleen, mesenteric lymph nodes $(\mathrm{MLN})$, and cervical lymph nodes (CLN) were harvested from the mice 7 days after the injection. The cells from the tissues were cultured for 2 days, and the levels of IgM were analyzed in the cell culture supernatants (Fig. 2A). The IgM amounts were increased in the culture supernatants of cells prepared from the CpG-DNA 1826-injected peritoneal fluid, but were not increased in the other tissues by CpG-DNA 1826 injection. To determine whether IgM is produced by CpG-DNA stimulation in vitro, the cells from the tissues were stimulated in vitro with PBS, CpG-DNA 1826 or non-CpG-DNA 2041 for 2 days and the levels of IgM were analyzed in the cell culture
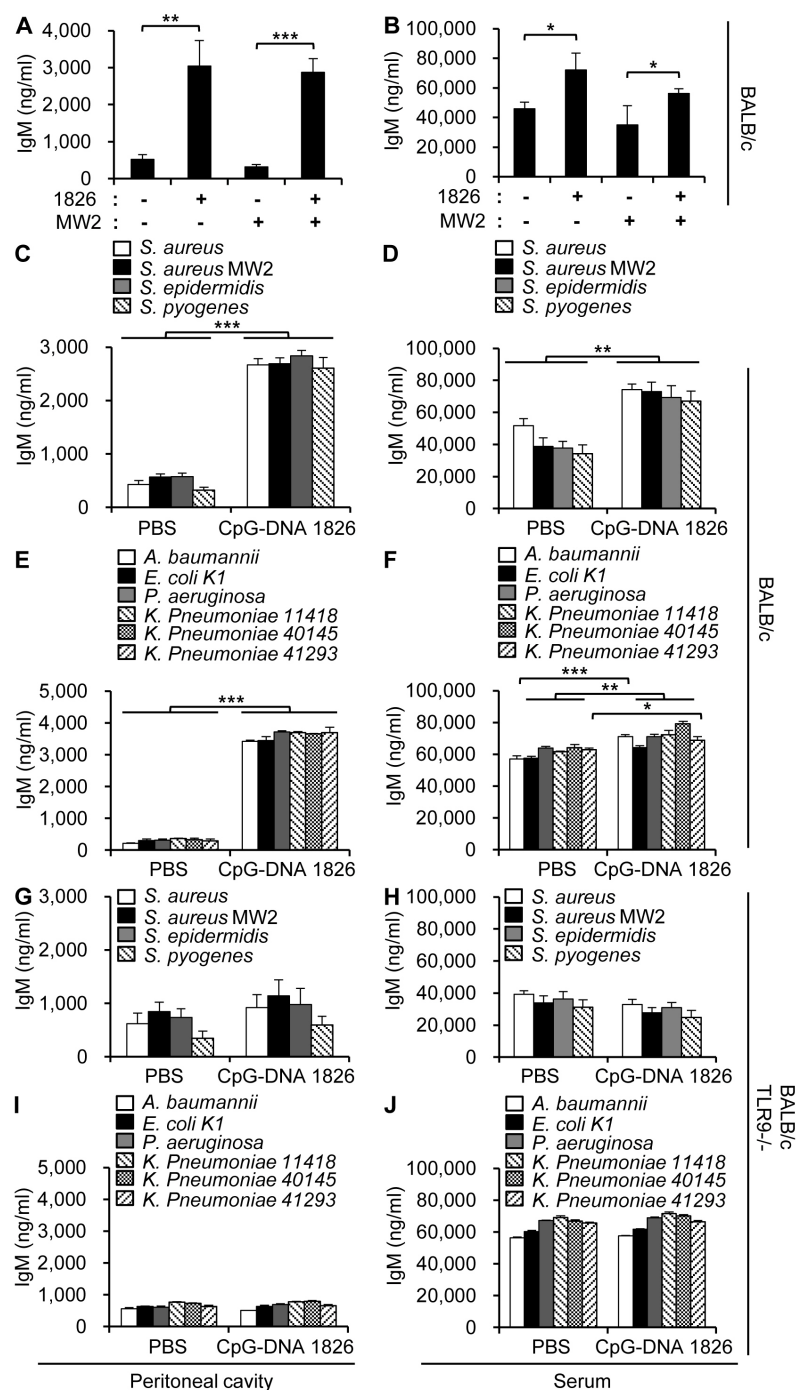

Fig. 1. Production of bacteria-reactive $\lg M$ in the mouse peritoneal cavity and serum by administration of CpG-DNA 1826. (A, B) $\mathrm{BALB} / \mathrm{C}$ mice were intraperitoneally injected with CpG-DNA 1826. After 7 days, the mice were intravenously injected with $S$. aureus MW2 $\left(1 \times 10^{7}\right.$ CFU). Peritoneal fluid and serum were collected from the mice two days after the bacterial infection. Bacteria-reactive IgM in the peritoneal fluid (A) and serum (B) was captured using $S$. aureus MW2-coated plates ( $\mathrm{n}=3 /$ group) and the concentrations of IgM were measured by ELISA. 1826, CpG-DNA 1826. MW2, S. aureus MW2. (C-F) BALB/C and (G-J) TLR9 $^{-1-}$ mice were intraperitoneally injected with CpG-DNA 1826. Seven days after the administration of CpG-DNA 1826, the peritoneal fluids (C, E, G, I) and serum $(\mathrm{D}, \mathrm{F}, \mathrm{H}, \mathrm{J})$ were collected. To measure the amounts of IgM reactive to bacteria, the indicated bacteria were added to poly-L-lysine-coated plates, and the amount of bound IgM was determined by ELISA ( $\mathrm{n}=3$ /group). The results presented are representative of three independent experiments. ${ }^{*} \mathrm{P}<0.05 ;{ }^{*} * \mathrm{P}<$ $0.005 ; * * * P<0.0005$. 

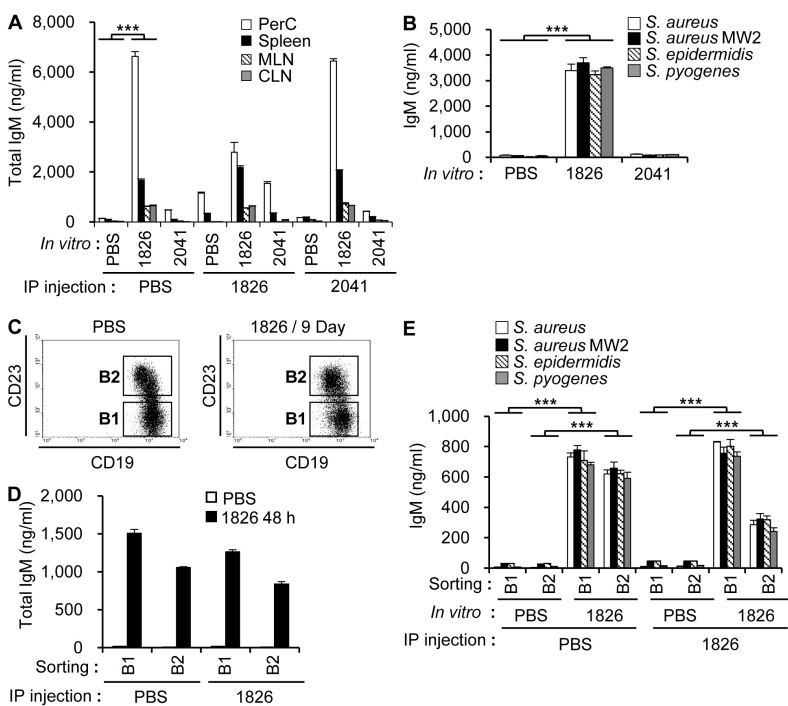

Fig. 2. Production of bacteria-reactive $\lg M$ in the mouse peritoneal cavity cells by CpG-DNA 1826 treatment in vitro. (A) BALB/C mice were intraperitoneally injected with PBS, CpG-DNA 1826, or non-CpG-DNA 2041. After 7 days, the cells from the peritoneal cavity (PerC), spleen, mesenteric lymph nodes $(\mathrm{MLN})$, and cervical lymph nodes $(\mathrm{CLN})$ were harvested and then treated with PBS, CpG-DNA 1826, or non-CpG-DNA 2041. The cell culture supernatants were collected after $48 \mathrm{~h}$, and the amounts of total IgM were determined by ELISA ( $n=3$ /group). (B) The peritoneal cells were harvested from PBS-injected mice, and the cells were treated with PBS, CpG-DNA 1826, or non-CpG-DNA 2041. The amounts of IgM reactive to the indicated Gram-positive bacteria in the culture supernatants were determined by ELISA ( $\mathrm{n}=3 /$ group). (C) B1 and B2 cells were isolated from PBS- or CpG-DNA 1826-injected peritoneal cavity using a FACSAria $^{\text {TM }}$ II system and fluorescence-labeled anti-mouse CD19 and anti-mouse CD23 antibodies. (D, E) Isolated B1 and B2 cells from the peritoneal cavity were stimulated with PBS or CpG-DNA 1826 for 4h. The cell culture supernatants were collected, and the amount of total IgM (D) and IgM reactive to Gram-positive bacteria (E) were determined by ELISA ( $n=3 /$ group). 1826, CpG-DNA 1826. 2041, non-CpG-DNA 2041. The results presented are representative of three experiments. $* * * P<0.0005$.

supernatants (Fig. 2A). The results show that the amounts of $\lg M$ were significantly increased in the peritoneal fluid, but only slightly increased in the other tissues by CpG-DNA 1826 stimulation in vitro compared to the PBS control or non-CpG-DNA 2041. When the CpG-DNA was pre-treated prior to the isolation of the peritoneal cells, the CpG-DNA-induced production of IgM was significantly reduced. To examine the bacteria-reactivity of the $\operatorname{lgM}$ produced by the CpG-DNA stimulation of the peritoneal cells in vitro, we measured the levels of bacteria-reactive $\lg M$ with the Gram-positive bacteria species. The levels of bacteria-reactive $\operatorname{IgM}$ were significantly increased by stimulation with CpG-DNA compared to PBS or non-CpG-DNA 2041 (Fig. 2B). To determine which B cells are associated with bacteria-reactive IgM secretion following
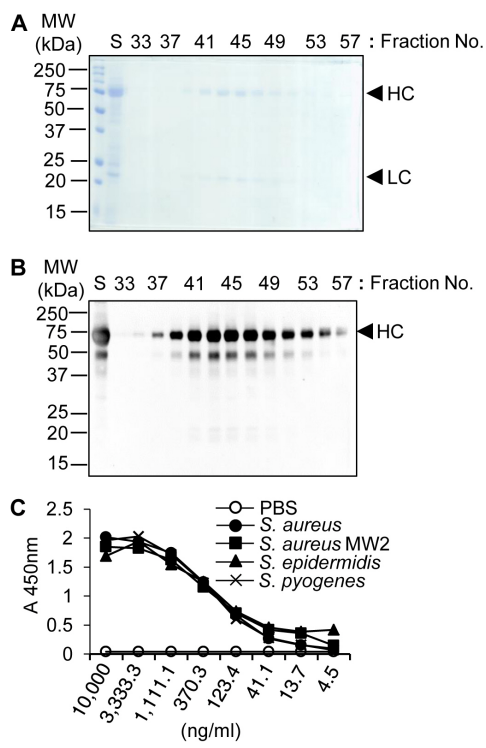

Fig. 3. Purification and characterization of monoclonal IgM antibody produced by CpG-DNA 1826 stimulation. (A, B) IgM-secreting hybridoma cells (clone 3F3F5) were cultured in Hybridoma-SFM for 5 days. The supernatants of the cell cultures were collected, and subjected to DEAE-sepharose column chromatography, and then, the protein fractions containing IgM were purified by gel filtration. The fractions containing monoclonal $\lg M$ antibody were analyzed by Coomassie brilliant blue staining (A) and Western blotting (B). S, cell culture supernatants; HC, heavy chain; LC, light chain. (C) The bacteria-reactivity of the purified IgM was measured by ELISA using plates coated with Gram-positive bacteria ( $n=3 /$ group). The results presented are representative of three experiments.

CpG-DNA stimulation, mice were intraperitoneally injected with the PBS control or CpG-DNA 1826. Nine days after the injection of CpG-DNA 1826, we sorted CD23 ${ }^{-} \mathrm{CD} 19^{+}$B cells (B1 cells) and $\mathrm{CD} 23^{+} \mathrm{CD} 19^{+} \mathrm{B}$ cells (B2 cells) from the peritoneal cavity lymphocytes (Fig. 2C). The sorted B cells were treated with CpG-DNA 1826 in vitro for 2 days, and the amounts of IgM in the supernatants of the cell cultures were measured by ELISA (Fig. 2D). The secreted amounts of IgM in response to CpG-DNA treatment were increased both in the B1 and B2 cells. However, the increase of IgM production by $B$ cells was reduced when the mice were pre-injected with CpG-DNA before the isolation of peritoneal cells, indicating that CpG-DNA influenced the $B$ cell activity in terms of antibody production (Fig. 2D). The amounts of bacteriareactive $\lg M$ in the $B$ cells were increased by CpG-DNA stimulation in vitro; however, the production of the bacteria-reactive IgM by the B2 cells was significantly reduced when CpG-DNA was pre-treated prior to the isolation of the peritoneal cells (Fig. 2E). The results suggest that bacteriareactive IgM was produced by the CpG-DNA treatment both in the B1 and B2 cells of the peritoneal cavity; however, there can be some difference in the regulation between the two 
subpopulations.

\section{Production of bacteria-reactive monoclonal IgM antibody} To obtain the bacteria-reactive monoclonal IgM antibody from the mouse peritoneal cavity cells, we screened the hybridoma cells secreting CpG-DNA-induced IgM. Seven days after the intraperitoneal injection of CpG-DNA 1826, the peritoneal cavity cells were harvested from the mice, and they were fused with myeloma SP2/0 cells, and screened in selective medium. After screening a hybridoma clone (3F3F5) secreting the bacteria-reactive monoclonal $\operatorname{lgM}$ antibody, the cells were cultured serum-free medium for 5 days. The bacteria-reactive monoclonal IgM antibody was purified from the culture supernatants using ion-exchange chromatography, followed by gel filtration chromatography. The IgM protein fractions were collected, loaded and run on a SDS-PAGE, and stained with Coomassie brilliant blue $\mathrm{R}$ solution (Fig. 3A). The fractions including the purified IgM were analyzed by Western blotting (Fig. 3B). We confirmed the ability of the purified monoclonal IgM antibody to bind several Gram-positive bacteria by ELISA (Fig. 3C).

\section{Bacteria-reactive monoclonal IgM antibody enhances phagocytosis of $S$. aureus MW2 in RAW 264.7 cells} To perform the phagocytosis assay in vitro, we selected murine macrophage cell line RAW 264.7. To investigate the phagocytosis efficacy of the bacteria-reactive monoclonal IgM antibody, we incubated fluorescein isothiocyanate (FITC)-labeled S. aureus MW2 with PBS, normal mouse IgM, or bacteriareactive monoclonal $\mathrm{IgM}$ antibody (3F3F5 IgM). The RAW 264.7 cells were treated with the antibody-bound $S$. aureus MW2, and then, phagocytosis was observed by confocal microscopy (Fig. 4A, B). The results indicate that the efficacy

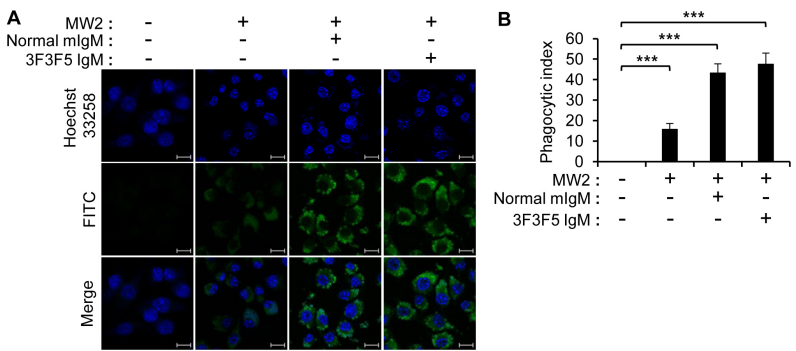

Fig. 4. Enhanced phagocytosis activity in RAW 264.7 cells by the bacteria-reactive monoclonal IgM antibody. FITC-labeled $S$. aureus MW2 cells $\left(3 \times 10^{8} \mathrm{CFU} / \mathrm{mL}\right)$ were incubated with PBS, normal mouse IgM (normal mlgM), or bacteria-reactive monoclonal IgM antibody (3F3F5 IgM) $(10 \mu \mathrm{g} / \mathrm{mL})$ for $1 \mathrm{~h}$ at $37^{\circ} \mathrm{C}$, and then added to RAW 264.7 cells in vitro. After $1 \mathrm{~h}$, the cells were washed with PBS, fixed, and stained with Hoechst No. 33258 to visualize the nuclei (blue). (A) Confocal images revealing phagocytosis of $S$. aureus MW2. Scale bars, $10 \mu \mathrm{m}$. MW2, S. aureus MW2. (B) The phagocytic index was analyzed ( $\mathrm{n}=5$ /group). The results presented are representative of three experiments. $* * * P<0.0005$. of phagocytosis was increased in the presence of the bacteria-reactive monoclonal IgM antibody or normal mouse IgM. However, there was no difference in phagocytic efficacy between the two IgM suggesting that the amounts of bacteria-reactive IgM were increased in the peritoneal cavity and sera following the injection of CpG-DNA 1826 (Fig. 1) but the phagocytic ability of the bacteria-reactive antibody was not enhanced by the administration of CpG-DNA 1826 compared to normal mouse IgM.

\section{DISCUSSION}

S. aureus has been recognized as a major cause of many infectious diseases including life-threatening diseases such as pneumoniae, sepsis, and endocarditis (19). Excessive use of multiple antibiotics to treat $S$. aureus infection has led to the emergence of methicillin-resistant staphylococcus aureus (MRSA) (20). As the efficacy of antibiotics decreases and the emergence of MRSA increases, a new strategy to replace antibiotics is urgently required. Several reports have suggested the development of vaccination and passive immunization using protective antibodies as an alternative strategy against MRSA infections (21-23).

In our previous experiments, we confirmed that CpG-DNA treatment enhanced the host protection following $S$. aureus infection, producing bacteria-reactive IgG antibodies which also have a protective role against infection (18). Here, we hypothesized that the CpG-DNA-induced production of antibodies, especially IgM, would be involved in the anti-bacterial effect of CpG-DNA. Because the levels of the bacteria-reactive $\operatorname{lgM}$ antibodies were significantly increased both in the peritoneal cavity and serum of mice following the administration of CpG-DNA (Fig. 1), we predicted that CpG-DNA triggered the activation of some B cell populations, producing bacteria-reactive IgM.

Natural antibodies, which exist without any exposure to antigens, have a broad antibacterial activity, and prevent several bacterial infections (24). Here, we confirmed that the amount of IgM produced by the CpG-DNA treatment was greatly increased in the peritoneal cavity of the mice and had a broad reactivity against several bacteria (Fig. 1). We suppose that the bacteria-reactive IgM has broad reactivity because the epitopes of the antibody might be common bacterial surface components such as carbohydrate, glycoprotein, and/or metabolites.

In addition, the secretion of $\operatorname{lgM}$ antibodies by the CpG-DNA treatment was significantly increased in the peritoneal cells when we isolated the peritoneal cells, spleen, MLN, and CLN and stimulated them with CpG-DNA in vitro (Fig. 2). It has been reported that most of the B1 cells were in the peritoneal cavity and produced broad bacterial-reactive antibodies against microbial agents $(25,26)$. We found that the bacteria-reactive $\lg M$ was produced both in the peritoneal $B 1$ and B2 cells, but higher levels of IgM were produced in the B1 cells than in the B2 cells (Fig. 2). 
Researchers have shown the efficacy of antibody-mediated phagocytosis against bacterial infection as a measure of the anti-bacterial effect in phagocytic cells $(24,27)$. When we examined the effects of the bacteria-reactive monoclonal IgM antibody (3F3F5 mlgM) against bacterial infection, the efficacy of phagocytosis against $S$. aureus MW2 infection in RAW 264.7 cells was enhanced by the monoclonal IgM antibody which was comparable to normal IgM (Fig. 4). Based on the results, we consider that $3 \mathrm{~F} 3 \mathrm{~F} 5 \mathrm{mlgM}$ could be a potent therapeutic agent against bacterial infection in emergency.

In conclusion, we proved that the bacteria-reactive IgM produced by CpG-DNA has protective roles against $S$. aureus infection by enhanced antibody-mediated phagocytosis in murine macrophage cell line. Despite numerous studies on the development of therapeutic antibodies against bacterial infection, no clinical antibodies have yet been approved. Therefore, future studies on infection therapy should include the development of a humanized antibody against MRSA infection, and this development might be useful to treat infectious diseases.

\section{MATERIALS AND METHODS}

\section{CpG-DNA administration}

CpG-DNA 1826 and non-CpG-DNA 2041, whose backbones of the sequences were modified with phosphorothioate, were synthesized by GenoTech Co. The following sequences were used: CpG-DNA 1826, 5'-TCCATGACGTTCCTGACGTT-3' and non-CpG-DNA 2041 as a negative control, 5'-CTGGTCTTTCTGGTTTTTTTCTGG-3'. The oligodeoxynucleotides were dissolved in distilled water, injected intraperitoneally in BALB/c mice or TLR9 ${ }^{-/-}$mice (50 $\mu \mathrm{g} /$ mouse).

\section{In vitro stimulation of mouse primary cells with CpG-DNA} Primary cells were harvested from the mice, homogenized, and re-suspended in RPMI 1640 medium containing 5\% FBS. After removing the erythrocytes, the cells were cultured in RPMI 1640 medium containing 5\% FBS with $100 \mathrm{U} / \mathrm{ml}$ of penicillin and $100 \mu \mathrm{g} / \mathrm{ml}$ of streptomycin. Next, each cell culture plate was treated with PBS or $5 \mu \mathrm{g} / \mathrm{ml}$ of CpG-DNA 1826 or $5 \mu \mathrm{g} / \mathrm{ml}$ of non-CpG-DNA 2041. After $48 \mathrm{~h}$, the cell culture supernatants were harvested and analyzed by ELISA to quantify the IgM levels.

\section{Sorting B cells from mouse peritoneal cells}

Peritoneal cells were stained with antibodies in sorting buffer (1 mM EDTA, 25 mM HEPES pH 7.0, 1\% FBS diluted in PBS). To isolate the $\mathrm{B}$ cells, the peritoneal cells were incubated with anti-mouse CD19 (BD Biosciences, San Jose, CA, USA) and anti-mouse CD23 (eBioscience, San Diego, CA, USA). To sort non- $B$ cells from the peritoneal cells, anti-mouse CD3 (BD Bioscience) was used to isolate T cells. B1 cells and B2 cells were sorted using a FACSAria ${ }^{\text {TM }}$ II system (Becton Dickinson Inc.).

\section{Purification of monoclonal IgM antibody}

IgM-secreting hybridoma cells (3F3F5 clone) were applied to the Hybridoma-SFM (Thermo Fisher Scientific Inc.), and grown at $125 \mathrm{rpm}$ in a shaker incubator containing $8 \% \mathrm{CO}_{2}$ at $37^{\circ} \mathrm{C}$. After 5 days, cell culture supernatants were obtained by centrifugation at 3,000 rpm for $10 \mathrm{~min}$, and purified using DEAE Sepharose ${ }^{T M}$ Fast Flow (GE Healthcare Co.) and HiPrep $^{\text {TM }}$ 16/60 Sephacryl ${ }^{\text {TM }}$ S-300 HR (Ge Healthcare Co.). The samples were loaded onto a SDS-PAGE, analyzed by Coomassie blue staining and Western blotting (28).

\section{Western blotting}

The purified IgM was resolved, and transferred onto nitrocellulose membranes, which were blocked with $5 \%$ Skim milk in PBS-T for $1 \mathrm{~h}$ at room temperature. Membranes were incubated with HRP-conjugated goat anti-mouse IgM ( $\mu$-chain specific) antibody (Merck Millipore Co.) for $2 \mathrm{~h}$ at room temperature. Immuno-reactive protein band intensities were measured with chemiluminescence reagent (Thermo Fisher Scientific Co.) as previously described (29).

\section{In vitro phagocytosis assays}

The mouse RAW 264.7 macrophage cell line was purchased from the American Type Culture Collection (ATCC). The cells were cultured overnight on poly-L-lysine-coated cover glass (Sigma-Aldrich Co.) in 12-well plates (Nunc Inc.). The FITC-labeled S. aureus MW2 was treated with PBS or IgM for 1 $\mathrm{h}$, and then, the bacteria were added to the plate cultured with RAW 264.7 cells. The cells were incubated for $1 \mathrm{~h}$ and fixed with $4 \%$ paraformaldehyde (Affymetrix Inc.). The fixed cells were washed with PBS, and then, Hoechst No. 33258 (Sigma-Aldrich Co.) was added at room temperature. The mounted cells were analyzed using a LSM 710 laser scanning microscope (Carl Zeiss Co.) previously described (18). The phagocytic index was measured by counting the number of FITC-labeled S. aureus MW2 phagocytosed by RAW 264.7 cells as previously described (18).

Additional Material and Methods are provided in the Supplementary Material.

\section{ACKNOWLEDGEMENTS}

This work was supported by grants from the National Research Foundation (2017R1A2B2007373, 2009-0093812) funded by the Ministry of Science and ICT in the Republic of Korea.

\section{CONFLICTS OF INTEREST}

The authors have no conflicting interests.

\section{REFERENCES}

1. Boes M (2000) Role of natural and immune IgM antibodies in immune responses. Mol Immunol 37, 
1141-1149

2. Casali P and Schettino EW (1996) Structure and function of natural antibodies. Curr Top Microbiol Immunol 210, 167-179

3. Hjelm F, Carlsson F, Getahun A and Heyman B (2006) Antibody-mediated regulation of the immune response. Scand J Immunol 64, 177-184

4. Ehrenstein MR and Notley CA (2010) The importance of natural IgM: scavenger, protector and regulator. Nat Rev Immunol 10, 778-786

5. Ricci ML, von Hunolstein C, Gomez MJ, Parisi L, Tissi L and Orefici G (1996) Protective activity of a murine monoclonal antibody against acute and chronic experimental infection with type IV group B streptococcus. J Med Microbiol 44, 475-481

6. Hustinx W, Benaissa-Trouw B, Van Kessel K et al (1997) Granulocyte colony-stimulating factor enhances protection by anti-K1 capsular IgM antibody in murine Escherichia coli sepsis. Eur J Clin Invest 27, 1044-1048

7. Connolly SE, Thanassi DG and Benach JL (2004) Generation of a complement-independent bactericidal IgM against a relapsing fever Borrelia. J Immunol 172, 1191-1197

8. Kinoshita M, Shinomiya N, Ono S et al (2006) Restoration of natural IgM production from liver $B$ cells by exogenous IL-18 improves the survival of burn-injured mice infected with Pseudomonas aeruginosa. J Immunol 177, 4627-4635

9. Schwartz JT, Barker JH, Long ME, Kaufman J, McCracken J and Allen LA (2012) Natural IgM mediates complementdependent uptake of Francisella tularensis by human neutrophils via complement receptors 1 and 3 in nonimmune serum. J Immunol 189, 3064-3077

10. Brown JS, Hussell T, Gilliland SM et al (2002) The classical pathway is the dominant complement pathway required for innate immunity to Streptococcus pneumoniae infection in mice. Proc Natl Acad Sci U S A 99, 16969-16974

11. Alugupalli KR, Gerstein RM, Chen J, Szomolanyi-Tsuda E, Woodland RT and Leong JM (2003) The resolution of relapsing fever borreliosis requires IgM and is concurrent with expansion of B1b lymphocytes. J Immunol 170, 3819-3827

12. Klinman DM, Yi AK, Beaucage SL, Conover J and Krieg AM (1996) CpG motifs present in bacteria DNA rapidly induce lymphocytes to secrete interleukin 6, interleukin 12, and interferon gamma. Proc Natl Acad Sci U S A 93, 2879-2883

13. Krieg AM, Yi AK, Matson $S$ et al (1995) CpG motifs in bacterial DNA trigger direct B-cell activation. Nature 374 , 546-549

14. Deng JC, Moore TA, Newstead MW, Zeng X, Krieg AM and Standiford TJ (2004) CpG oligodeoxynucleotides stimulate protective innate immunity against pulmonary Klebsiella infection. J Immunol 173, 5148-5155
15. Ishii KJ, Ito S, Tamura $\mathrm{T}$ et al (2005) CpG-activated Thy1.2 + dendritic cells protect against lethal Listeria monocytogenes infection. Eur J Immunol 35, 2397-2405

16. Mohamed W, Domann E, Chakraborty T et al (2016) TLR9 mediates $\mathrm{S}$. aureus killing inside osteoblasts via induction of oxidative stress. BMC Microbiol 16, 230

17. Wu HM, Wang J, Zhang B, Fang L, Xu K and Liu RY (2016) CpG-ODN promotes phagocytosis and autophagy through JNK/P38 signal pathway in Staphylococcus aureus-stimulated macrophage. Life Sci 161, 51-59

18. Kim TH, Kim D, Gautam A et al (2018) CpG-DNA exerts antibacterial effects by protecting immune cells and producing bacteria-reactive antibodies. Sci Rep 8, 16236

19. Adem PV, Montgomery CP, Husain AN et al (2005) Staphylococcus aureus sepsis and the WaterhouseFriderichsen syndrome in children. N Engl J Med 353, 1245-1251

20. Neu HC (1992) The crisis in antibiotic resistance. Science $257,1064-1073$

21. Schaffer AC and Lee JC (2008) Vaccination and passive immunisation against Staphylococcus aureus. Int J Antimicrob Agents 32 Suppl 1, S71-78

22. Fattom Al, Horwith G, Fuller S, Propst $M$ and Naso R (2004) Development of StaphVAX, a polysaccharide conjugate vaccine against $\mathrm{S}$. aureus infection: from the lab bench to phase III clinical trials. Vaccine 22, 880-887

23. Vernachio J, Bayer AS, Le T et al (2003) Anti-clumping factor A immunoglobulin reduces the duration of methicillin-resistant Staphylococcus aureus bacteremia in an experimental model of infective endocarditis. Antimicrob Agents Chemother 47, 3400-3406

24. Zhou ZH, Zhang Y, Hu YF, Wahl LM, Cisar JO and Notkins AL (2007) The broad antibacterial activity of the natural antibody repertoire is due to polyreactive antibodies. Cell Host Microbe 1, 51-61

25. Hoffman W, Lakkis FG and Chalasani G (2016) B Cells, Antibodies, and More. Clin J Am Soc Nephrol 11, 137-154

26. Cole LE, Yang Y, Elkins KL et al (2009) Antigen-specific B-1a antibodies induced by Francisella tularensis LPS provide long-term protection against $\mathrm{F}$. tularensis LVS challenge. Proc Natl Acad Sci U S A 106, 4343-4348

27. Panda S, Zhang J, Tan NS, Ho B and Ding JL (2013) Natural IgG antibodies provide innate protection against ficolin-opsonized bacteria. EMBO J 32, 2905-2919

28. Park BK, Maharjan S, Lee SI et al (2018) Generation and characterization of a monoclonal antibody against MERSVoV targeting the spike protein using a synthetic peptide epitope-CpG-DNA-liposome complex. BMB Rep 52, 397402

29. Kim DW, Shin MJ, Choi YJ et al (2018) Tat-ATOX1 inhibits inflammatory responses via regulation of MAPK and NF-kB pathways. BMB Rep 51, 654-659 